\title{
Dendritic Cell Targets for Self-Replicating RNA Vaccines
}

\author{
Kenneth C. McCullough ${ }^{*}$ Panagiota Milona, Thomas Démoulins, Pavlos Englezou and Nicolas Ruggli \\ Institute of Virology and Immunology, 3147 Mittelhaeusern, Switzerland \\ *Corresponding author: Kenneth McCullough, Institute of Virology and Immunology, Sensemattstrasse 293 , $\mathrm{CH}-3147 \mathrm{Mittelhäusern,} \mathrm{Switzerland,} \mathrm{Tel:}$ \\ +41-31-848-9262; E-mail: kenneth.mccullough@vetsuisse.unibe.ch
}

Rec date: November 25, 2014, Acc date: January 20, 2015, Pub date: January 30, 2015

Copyright: (c) 2015 McCullough KC, et al. This is an open-access article distributed under the terms of the Creative Commons Attribution License, which permits unrestricted use, distribution, and reproduction in any medium, provided the original author and source are credited.

\section{Mini Review}

Keywords: Dendritic cells; Replicon RNA; Self-replicating vaccines; Nano particulate delivery

\section{Advances in Vaccine Development}

Vaccination is the cornerstone for controlling many pathogen infections [1-8], and is also under scrutiny for cancer Immunoprophylaxis/immunotherapy $[9,10]$. Induction of both antibody and cell-mediated immune (CMI) defences is preferable for ensuring robust immune defence against most pathogen infections, although defence against certain pathogens may require a more dominant CMI response as exemplified by hepatitis $\mathrm{C}$ virus [11]. A major drawback of most current vaccines is their non-replicative nature $[3,8]$, being inactivated or recombinant protein-based vaccines, limiting the amount of antigen available to the immune system. Replicating vaccines offer several rounds of antigen production to increase the efficacy of immune defence induction [3,8]. This replicative nature can mimic the situation with replicating pathogens, whereby antigen can be directed into both MHC Class I and MHC Class II presentation pathways, thus promoting humoral and CMI defences. These characteristics are also important for cancer vaccines, particularly when the immunogen in question is weak.

Live, attenuated vaccines offer the properties of a replicating vaccine, but may suffer from drawbacks such as potential reversion or recombination to virulence, production problems, and potentially high biosafety precautions for their generation. Moreover, it may not be possible to vaccinate all recipients at risk, for example young children. In addition, live, attenuated vaccines for cancer immunotherapy may not prove applicable, even when employed as vector vaccines carrying the cancer antigen. Vector vaccines can suffer from similar production problems as live, attenuated vaccines, and both forms of vaccine may prove inefficient in the face of an existing immunity against the vector.

Nucleic acid-based vaccines can circumvent many of the problems associated with both inactivated and live, attenuated or vector vaccines $[3,8,12-16]$. DNA-based vaccines have a proven success, but often in murine models [8]. A major obstruction lies with DNA vaccines having to overcome the resistance of the nuclear membrane particularly problematic with the major antigen-presenting cell type of the body, the dendritic cell (DC), unless a nuclear targeting entity is employed. Moreover, DNA retains certain notoriety for persistence within cells, and potential biosafety risks of recombination with host cell chromosomes. Such difficulties do not arise with RNA vaccines, but these suffer from high lability, especially in RNase-containing extracellular and intracellular environments. Delivery of DNA vaccines has provided the means of facilitating RNA vaccine applicability, namely through the employment of biodegradable, nanoparticulate delivery vehicles $[2,8,17-21]$. The delivery vehicle can protect the RNA as well as enhancing its delivery to cells, for which interaction with DCs would prove a major contribution to development of immune defences. Figure 1 summarises main elements contained in the present review below, including the advantages offered when applying biodegradable, nanoparticulate vehicles for delivery of self-amplifying replicon RNA to DCs.

\section{Delivery to Dendritic Cells}

Vaccine delivery to DCs is an important consideration due to their essential roles in immune defence development [8,17,19,21-24] - DCs are referred to as the "professional antigen-presenting cells". A major advance in this area came from the application of mRNA vaccines. An interesting mode of delivery was the in vitro pulsing of isolated DCs with RNA vaccine prior to re-injection in vivo $[18,20,25,26]$. This proved applicable with mRNA molecules encoding the antigen of choice, or mRNA from the tumour cell against which vaccination was desired. Moreover, RNA pulsing offered the means of controlling the environment during delivery to the DCs, important considering the high lability of RNA to RNase degradation.

While the RNA pulsing approach has value for cancer immunotherapy, it is not the first choice for vaccination against infectious diseases. On the contrary, RNA pulsing of DCs in vitro can provide important details on the relative efficacy of the delivery under investigation $[8,20]$. In this context, application of biodegradable nanoparticle delivery systems - have been widely studied and applied with protein-based vaccines - has demonstrated their high potential for nucleic acid delivery to DCs $[8,20]$. Importantly, nanoparticulate formulation can both protect an RNA vaccine and promote delivery to the DCs $[8,19-21]$. Nevertheless, mRNA vaccines can be generated with applicability as "naked" RNA (no delivery vehicle). Such approaches require that the mRNA be protected from RNase degradation, for example by 5' capping and/or addition of a poly-A tail at the 3 ' end, or addition of protamine [20,27-30]. An alternative to the capping strategy has employed large quantitates of the mRNA vaccines [20,31]. As such, these are more likely to prove inefficient when encoding weakly immunogenic antigens, which can be the case with tumour antigens. Moreover, the efficiency of mRNA interaction with DCs remains a point of discussion, due to the reported poor uptake of "naked" mRNA by DCs [32].

RNA vaccine development has found a clear level of success, with most vaccines based on the mRNA principle $[8,20,27,33]$. A substitute to the employment of "naked" mRNA or high concentrations, and thus circumventing their inherent restrictions, comes from the field of biodegradable delivery vehicle application. Nanoparticulate vehicles, which have involved primarily lipid-based and carbohydrate-based systems $[8,20]$, can enhance the protection of the RNA, or even render the capping procedure unnecessary. 


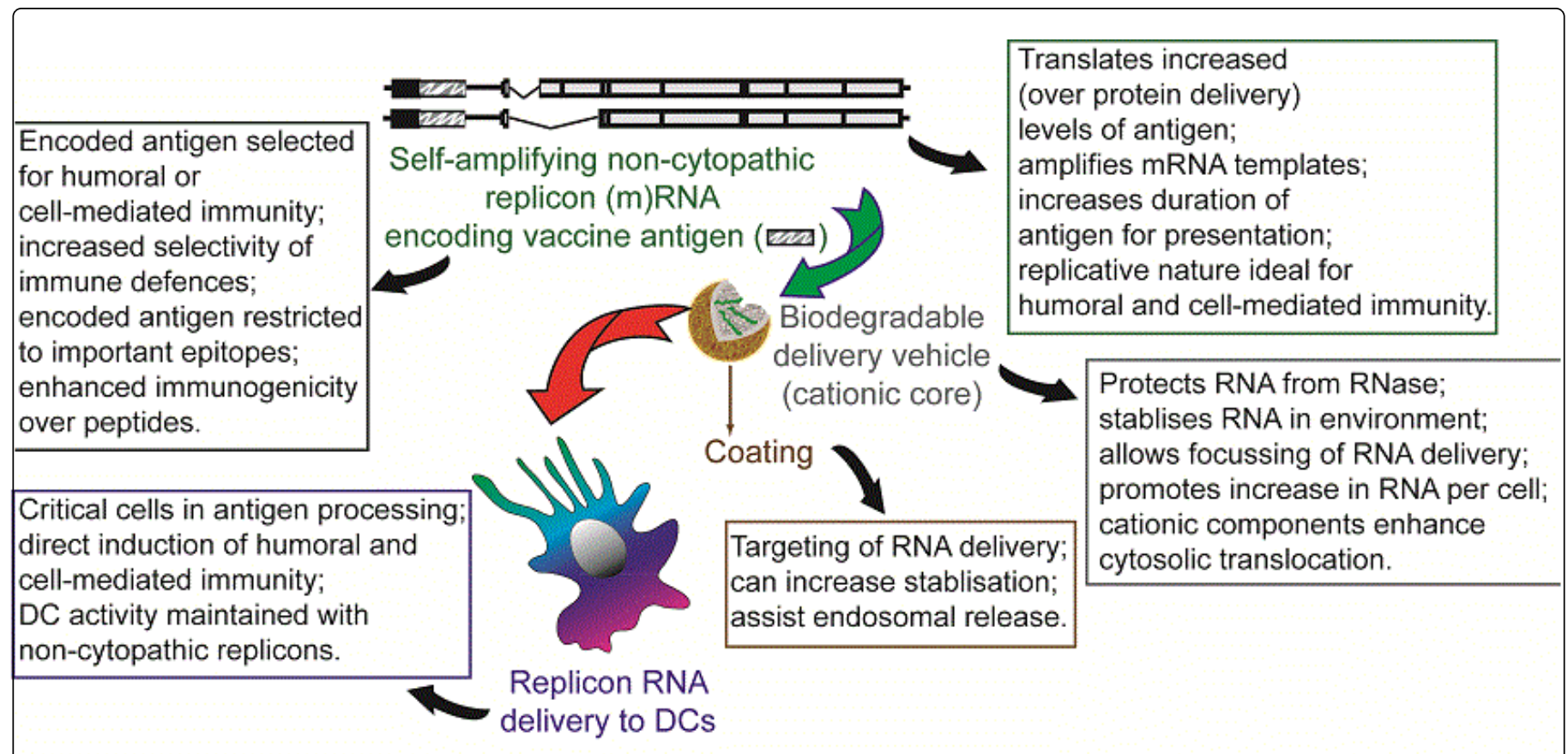

Figure 1: Summary of the critical components to consider when creating self-amplifying replicon RNA vaccines for delivery to DCs by biodegradable nanoparticulate vehicles. For each of these components, the advantages offered are shown in the corresponding boxes.

Moreover, many of these delivery vehicles have a proven capacity for interacting with DCs for delivery of their cargoes; in the case of RNA delivery, this has also led to the end goal of the delivery translation of the encoded vaccine antigen [3,8,19-21].

\section{Self-Amplifying RNA vaccines}

Vaccines based on classical mRNA molecules, similar in structure to the mRNA molecules found within cells, can have a clear efficacy. Nevertheless, they encode for a single protein, and are non-replicating entities. As such, the mRNA-derived antigen available to the immune system remains limited to that translated during the lifetime of the mRNA in the cell. Elaboration of the RNA vaccine approach has led to the development of vaccines based on larger RNA molecules encoding for several proteins in addition to the vaccine antigen, and therefore more complex in structure and nature than the mRNA molecules. These are the RNA molecules derived from replicon technology [34-37], possessing a replicative or self-amplifying capacity, which relates to their derivation from defective virus genomes. Not only do they encode for the vaccine antigen of choice, but also the endogenous viral proteins necessary for the replication and therefore amplification of the RNA within the cells. Consequently, the replicon RNA inherits the parent genome capacity for efficient replication and translation, but will not produce progeny virus due to the absence of genes required for construction and maturation of the virus infectious particle -so-called structural genes. For these reasons, replicon RNA vaccines are biosafe, and readily engineered to carry genes encoding the desired vaccine antigens [34-37].

Self-amplifying replicon RNA are derived from what are termed "positive strand viruses" and "negative strand viruses" [3,8,38,39]. The former class of virus carries a genome with a ribosomal entry site at its 5 ' end, which can thus function as a mRNA to initiate translation, including its own replication machinery (polymerase complex proteins, which are non-structural). Negative strand viruses, and therefore their replicons, require delivery together with their polymerase (consequently, a structural protein) to initiate replication for synthesis of the positive strand mRNA molecules required for translation. Both positive strand and negative strand replicon RNA vaccines have been employed, based on virus replicon particles (VRP). These VRPs are produced in cell lines providing the deleted structural gene in trans for the generation of virus particles carrying replicons; VRP vaccines have proven efficacy [3,8,34-39].

With replicons derived from negative strand viruses, VRP-based delivery remains the method of choice. This is due to the availability in the VRP structure of the polymerase essential for the negative strand replicon to produce mRNA templates for translation of the encoded proteins. In contrast, the positive strand replicon RNA itself acts as a mRNA, promoting translation of the encoded antigens and its replication machinery to increase the number of mRNA templates. As such, positive strand replicons are more readily adaptable to delivery by biodegradable, delivery vehicles [8]. Indeed, replicons derived from positive strand viruses have been referred to as "self-amplifying mRNA". This is somewhat misleading, because they should not be confused with mRNA molecules found in the cell, or with the mRNA vaccines that are non-replicating. Firstly, replicon RNA is a much larger molecule than classical mRNA molecules, and therefore requires different considerations in terms of its stability and structure, particularly with respect to delivery into cells. Secondly, replicon RNA encodes for a number of proteins, whereas classical mRNA molecules encode a single protein. The replicon RNA will produce the antigen of interest from a vaccine point of view, as well as the proteins required for replicon replication and therefore amplification of the number of RNA molecules to function as mRNAs. 
With their mimicking of the parent virus genome, the replicon RNA replication together with expanded intracellular translation of the antigens favour antigen processing via both MHC Class I and MHC Class II [8]. This, in turn, can induce both humoral and cellular immune responses, as well as providing a longer-lasting antigen expression compared to non-replicating mRNA. Together, these outcomes provide the stage for a more robust immune defence development.

With the RNA-based vaccine now possessing a replicating characteristic, it has been referred to as self-amplifying, selfreplicating, or just simply replicon RNA vaccine $[3,8]$. There is the notion that these can be termed self-amplifying or self-replicating mRNA vaccines, but this is only applicable when derived from a positive strand virus, with which the genome can function as an mRNA. However, the self-replicating RNA should not be confused with the mRNA molecule encoding for one antigen. Self-replicating RNA is a more complex structure encoding for a number of genes the vaccine antigen together with all the proteins associated with the replicative machinery of the viral genome from which the replicon was derived. Therefore, the term mRNA for such self-replicating RNA entities is somewhat erroneous, especially considering that the more appropriate descriptive term of replicon RNA has been employed for two decades [34-37].

\section{Important Considerations for Replicon RNA Vaccine Delivery}

One of the most important considerations for applying nanoparticulate vehicle delivery to dendritic cells is the type of replicon RNA mentioned above, in terms of being derived from a positive strand versus negative strand virus [38]. Clearly, the highest potential for application of biodegradable delivery vehicles is with positive strand replicon RNA, due to their capacity for functioning as a mRNA [8].

A second important consideration is delivery to the all-important DCs. Much of the work on positive strand replicon vaccines has applied RNA derived from Alphaviruses [34,35,37], primarily using the aforementioned approach of VRP-based delivery. However, VRPs behave like the parent virus in terms of targeting, and cannot be manipulated for focussed delivery to DCs [8]. Moreover, VRPs can be species-restricted, and encumbered by pre-existing immunity against the virus (and therefore the VRP). Biodegradable particle delivery overcomes both of these hurdles, facilitating controlled delivery to DCs of many species including humans.

The third important consideration, which the delivery vehicle cannot modify, is the biological nature of the replicon. Alphavirus replicons, like their parent viruses, are known to be cytopathic. Destruction of the targeted cell would be problematic for DCs, which display characteristics of slow processing and retention of antigen for prolonged interaction with the adaptive immune system. Accordingly, DCs would benefit from vaccine delivery utilising non-cytopathogenic replicon RNA, such as that derived from the Flavivirus Kunjin virus and the Pestivirus classical swine fever virus $[8,36,40]$.

A third important consideration is that Alphavirus genomes must be 5 '-capped to ensure ribosomal entry for translation [41], which also protects the RNA. By inference to the role of capping with mRNA vaccines, the capping of Alphavirus replicons should enhance their applicability without the need for a delivery vehicle. Flaviviruses also require 5 -capping to promote both polyprotein translation and protection of the RNA [42], although an uncapped flavivirus vaccine has been developed [43]. In contrast, application of Pestivirus replicon RNA circumvents the necessity to provide a capping step in the construction and production of the vaccine [40,44], due to the existence of the ribosomal entry site in the 5'-UTR (Figure 2). Construction of replicon RNA vaccines without recourse to capping procedures would have clear advantages for cell-free production of the vaccines on a commercial scale.

The final important consideration is the insertion into the RNA sequence of the "gene of interest" encoding the desired vaccine antigen (Figure 2, GOI). This figure shows the non-cytopathic Pestivirus genome, and two forms of replicon that have been generated $[2,45,46]$. These are replicons lacking a single structural glycoprotein, and replicons lacking all structural glycoproteins. Both are perfectly viable as vaccine vectors, but the latter is the more reliant on biodegradable nanoparticulate delivery. This is due to the requirements of VRP formation, in which the deleted gene has to be supplied in trans in a complementing cell line, more readily achievable when one gene is lacking rather than several. In the example shown, NotI endonuclease restriction sites are inserted at the $3^{\prime}$ end of the Npro leader autoprotease of the replicon RNA, which facilitates introduction of the gene of interest (GOI). This GOI can encode for a pathogen antigen or a tumour antigen. The advantage therein is that the translated antigen would be in its natural conformation, which is important when conformational epitopes are required for eliciting the desired immune responses. This is more critical for inducing humoral immunity than cellular immunity. Indeed many epitopes important for cellular immune defences - both helper and cytotoxic T-lymphocytes - are sequential rather than conformational. Accordingly, one may also introduce nucleotide sequences encoding epitopes rather than complete antigens. This still offers the advantages of the selfamplifying replicon RNA vaccines, as summarised in Figure 1. Moreover, the use of replicon RNA to encode peptide antigens will prove far superior to attempts with the peptide antigens themselves, particularly when the latter lack in potency. The replicon-encoded peptide antigens can also prove of high value when an antigen is difficult to purify, or is actually toxic when delivered to cells. By avoiding the generation of such antigens, through encoding for the peptide epitope, the replicon RNA vaccine has further added potential.

\section{Nanoparticulate Vehicle Delivery of Self-Amplifying RNA to Dendritic Cells}

Delivery of self-amplifying replicon RNA vaccines by biodegradable particles was first proposed in 2008 [40]. Nanoparticulate delivery vehicles offer clear advantages for delivery to DCs, whether that be protein-based antigens, DNA or RNA $[8,20,47,48]$. Indeed, application of polysaccharide, lipid and lipoplex technologies is showing high potential for replicon RNA delivery [2,40,45,49,50]; cylindrical BSAbased particle delivery have also been reported [51]. The success of synthetic, biodegradable chitosan-based nanogels for protein and DNA delivery to DCs $[52,53]$ was elaborated to display an efficient capacity for transporting replicon RNA into DCs, leading to RNA translation in vitro and immune responses induction in vivo [2]. Lipoplex formulations are also proving efficient for replicon RNA delivery to DCs, facilitating both translation and immune response induction in vivo [45]. Importantly, the replicon RNA was shown to associate physically with these delivery vehicles, confirming the role of the nanoparticulate systems in the actual delivery of the RNA to the DCs. This was important, because the replicon RNA - derived from 
the non-cytopathogenic Pestivirus classical swine fever virus - was non-infectious, incapable of entering the DCs or inducing an immune response in vivo without the assistance of the delivery vehicle $[2,45]$.

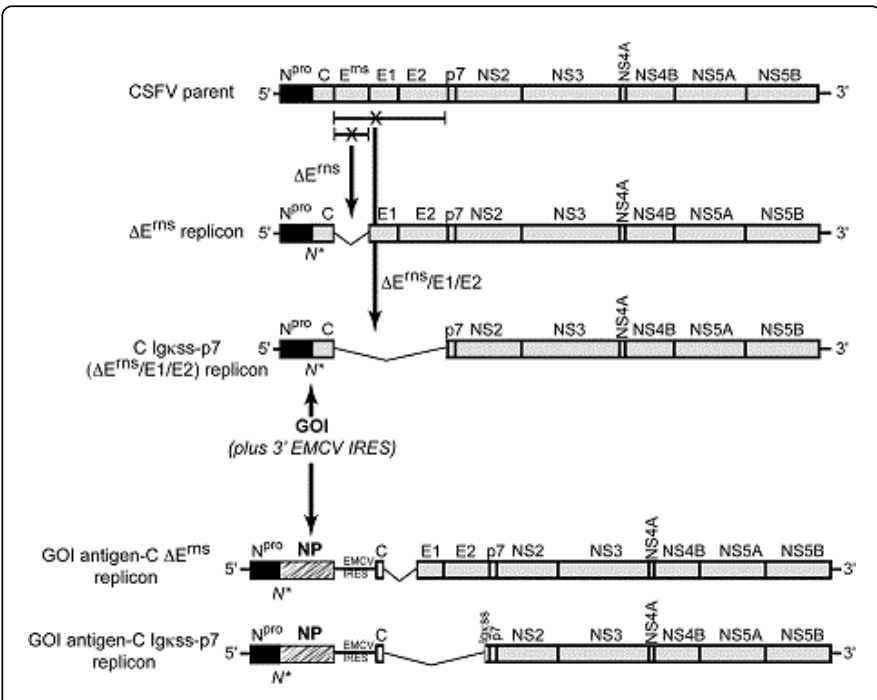

Figure 2: Schematic representation of the non-cytopathic pestivirus, classical swine fever virus (CSFV), genome. Two examples are shown for the types of replicon RNA which can be generated $\Delta$ Erns replicon lacking a single (Erns) gene, and C-Igkss-p7 replicon lacking all three structural glycoproteins. The NotI endonuclease restriction site introduced to facilitate insertion of genes encoding the vaccine antigen, are shown at the 3' end of the Npro leader autoprotease as $\mathrm{N}^{*}$. The site of the gene of interest (GOI) encoding the vaccine antigen or epitope is represented by the hashed box; it is followed by an additional insertion - an EMCV IRES - to restart the translation. This IRES is required, because translation terminates after the GOI. Normally, the Pestivirus genome is translated as single polyprotein, cleaved into individual proteins by autoprotease activities; with the insertion of the vaccine GOI, translation is terminated after the vaccine antigen is translated, and must be restarted to translate the proteins for the replicon translation.

Although Alphavirus replicon RNA application was reported with lipid-based formulations [50], no interaction with DCs was demonstrated. Moreover, these are both cytopathogenic replicons and capable of inducing immune responses as "naked" RNA in the absence of a delivery vehicle. Importantly, no physical association of the replicon RNA with the lipid-based formulations was shown, just an interference of the lipids in the assay to detect RNA. Considering that the unformulated Alphavirus RNA was already a functional vaccine, albeit relatively inefficient, the employed lipid formulations may not be delivering the RNA, but acting more as an adjuvant. Indeed, this replicon RNA was co-formulated with pre-formed squalene-based nanoemulsions [54], employing the squalene-based MF59 adjuvant [55]. Nonetheless, cell delivery of replicon RNA by nanoparticulate delivery vehicles [40] has clearly been demonstrated [2,45,51], leading to translation and replication in DCs and the induction of immune responses in vivo.

Although nanoparticulate vehicle delivery of replicon RNA as selfamplifying mRNA was first proposed in 2008 [40], progress has involved few groups, with evidence of in vivo applicability appearing only during the past 3 years $[2,45,50]$. While this pre-clinical evaluation shows high promise, clinical trial advancement is at an early stage $[39,56]$ and there are as yet no products on the market, although the UniVax consortium (www.univax-fp7.eu) is pursuing a clinical trial effort within its project tasks [57]. In this context, reference can be taken from the reports on clinical trials with non-replicating mRNA vaccines. These are often delivered as "dendritic cell vaccines" whereby the DCs have been transfected with the mRNA [8,58-64], but mRNA vaccines have been employed for direct vaccination $[62,64,65]$ and nanoparticulate delivery [59]. Such approaches were also applied in fields other than cancer vaccination [8,59], for example against HIV [58] and influenza [27]. Importantly, self-amplifying replicon RNA vaccines have been trialled as VRPs [56,66-69], which demonstrates the high potential for these RNA vaccines when delivered by nanoparticulate vehicles to DCs.

\section{Concluding Remarks on Self-Amplifying RNA Delivery to Dendritic Cells}

New generation RNA vaccines can be derived from defective viral genomes - the self-amplifying or self-replicating replicon RNA. These lend themselves readily to encoding for vaccine antigens of choice, and offer high potential for weakly immunogenic antigens, such as tumour antigens. In this context, important characteristics are the multiple rounds of translation and amplification from the RNA replication, which the replicon offers. Current molecular biology techniques allow for production of replicon RNA under cell-free conditions, which is important considering the larger and more complex nature of replicon RNA compared with mRNA.

Application of synthetic, biodegradable nanoparticulate delivery vehicles provides for both protection of the RNA and its delivery to the DCs, all-important for inducing efficient immune defence development. Employing non-cytopathic replicon RNA ensures that DCs are capable of maintaining antigen synthesis for a prolonged period, increasing the chances of inducing a robust immune defence. Moreover, with the replicon RNA translation in the DCs, the antigen is produced within the cell capable of priming the development of both humoral and cell-mediated immune defences. By such means, the whole process provides a novel synthetic vaccine, which could reduce vaccine production costs and time for manufacture, important in emergency situations such as during influenza pandemics. The characteristics also offer advantages when the encoded antigen is weakly immunogenic, as in the case of tumour antigens, or requires an immune response development more in favour of cell-mediated defences.

One important characteristic of the chitosan-based and lipoplexbased delivery vehicles, which will increase future developments of the technology, is their cationic composition. On the one side, this facilitates encapsulation of the replicon RNA. In turn, this cationic nature may prove advantageous following accumulation in vesicular bodies such as macropinosomes or caveolar endocytic vesicles. As the early endosomes come into play, these vesicular bodies begin to acidify. Thereby, it is essential that the intra-vesicular environment favour vesicular membrane destabilisation leading to efficient cytosolic translocation of the RNA [8]. Such a delivery step is critical following delivery of self-amplifying replicon RNA to DCs - the RNA must be transported to the site for replicon RNA translation, and avoid the degradative nature of the maturing endosomal system. It is also important that the formulation permits efficient decompaction of the RNA from the delivery vehicle, to facilitate ribosomal entry and 
subsequent interaction of the translated polymerase proteins. Our current studies are increasing the understanding of such events, ensuring that the delivery of the replicon RNA vaccines will provide end-goals of replicon RNA translation and replication that are at their most efficient.

\section{References}

1. Combadiere B, Liard C (2011) Transcutaneous and intradermal vaccination. Hum Vaccin 7: 811-827.

2. McCullough KC, Bassi I, Milona P, Suter R, Thomann-Harwood L, et al. (2014) Self-replicating Replicon-RNA Delivery to Dendritic Cells by Chitosan-nanoparticles for Translation In Vitro and In Vivo. Mol Ther Nucleic Acids 3: e173.

3. McCullough KC, Demoulins T, Milona P, Suter R, Thomann-Harwood L, et al. (2014) Self-amplifying replicon RNA vaccine delivery to dendritic cells by synthetic nanoparticles. Vaccines 2: 735-754

4. Pulendran B, Ahmed R (2006) Translating innate immunity into immunological memory: implications for vaccine development. Cell 124 849-863.

5. Randolph GJ, Jakubzick C, Qu C (2008) Antigen presentation by monocytes and monocyte-derived cells. Curr Opin Immunol 20: 52-60.

6. Shortman K, Lahoud MH, Caminschi I (2009) Improving vaccines by targeting antigens to dendritic cells. Exp Mol Med 41: 61-66.

7. Ueno H, Klechevsky E, Schmitt N, Ni L, Flamar AL, et al. (2011) Targeting human dendritic cell subsets for improved vaccines. Semin Immunol 23: 21-27.

8. McCullough KC, Bassi I, Démoulins T, Thomann-Harwood LJ, Ruggli N (2012) Functional RNA delivery targeted to dendritic cells by synthetic nanoparticles. Ther Deliv 3: 1077-1099.

9. Danhier F, Ansorena E, Silva JM, Coco R, Le Breton A, et al. (2012) PLGA-based nanoparticles: an overview of biomedical applications. Control Release 161: 505-522.

10. Klippstein R, Pozo D (2010) Nanotechnology-based manipulation of dendritic cells for enhanced immunotherapy strategies. Nanomedicine 6: 523-529.

11. Abdel-Hakeem MS, Shoukry NH (2014) Protective immunity against hepatitis C: many shades of gray. Front Immunol 5: 274.

12. Dow SW, Fradkin LG, Liggitt DH, Willson AP, Heath TD, et al. (1999) Lipid-DNA complexes induce potent activation of innate immune responses and antitumor activity when administered intravenously. J Immunol 163: 1552-1561.

13. Li W, Szoka FC Jr (2007) Lipid-based nanoparticles for nucleic acid delivery. Pharm Res 24: 438-449.

14. Liu MA (2010) Gene-based vaccines: Recent developments. Curr Opin Mol Ther 12: 86-93.

15. Markov OO, Mironova NL, Maslov MA, Petukhov IA, Morosova NG, et al. (2012) Novel cationic liposomes provide highly efficient delivery of DNA and RNA into dendritic cell progenitors and their immature offsets. J Control Release 160: 200-210.

16. Wasungu L, Hoekstra D (2006) Cationic lipids, lipoplexes and intracellular delivery of genes. J Control Release 116: 255-264.

17. Romani N, Thurnher M, Idoyaga J, Steinman RM, Flacher V (2010) Targeting of antigens to skin dendritic cells: possibilities to enhance vaccine efficacy. Immunol Cell Biol 88: 424-430.

18. Boczkowski D, Nair S (2010) RNA as performance-enhancers for dendritic cells. Expert Opin Biol Ther 10: 563-574.

19. Cruz LJ, Tacken PJ, Rueda F, Domingo JC, Albericio F, et al. (2012) Targeting nanoparticles to dendritic cells for immunotherapy. Methods Enzymol 509: 143-163.

20. Garg N, Dwivedi P, Prabha P, Tyagi RK (2013) RNA pulsed dendritic cells: an approach for cancer immunotherapy. Vaccine 31: 1141-1156.

21. Hamdy S, Haddadi A, Hung RW, Lavasanifar A (2011) Targeting dendritic cells with nano-particulate PLGA cancer vaccine formulations. Adv Drug Deliv Rev 63: 943-955.
22. Belizaire R, Unanue ER (2009) Targeting proteins to distinct subcellular compartments reveals unique requirements for $\mathrm{MHC}$ class I and II presentation. Proc. Natl. Acad. Sci. 106: 17463-17468.

23. Kawasaki N, Rillahan CD, Cheng TY, Van Rhijn I, Macauley MS, et al. (2014) Targeted delivery of mycobacterial antigens to human dendritic cells via Siglec-7 induces robust $\mathrm{T}$ cell activation. J Immunol 193 : 1560-1566.

24. Sehgal K, Dhodapkar KM, Dhodapkar MV (2014) Targeting human dendritic cells in situ to improve vaccines. Immunology Letters. Immunology Letters 162: 59-67.

25. Naka T, Iwahashi M, Nakamura M, Ojima T, Nakamori M, et al. (2008) Tumor vaccine therapy against recrudescent tumor using dendritic cells simultaneously transfected with tumor RNA and granulocyte macrophage colony-stimulating factor RNA. Cancer Sci 99: 407-413.

26. Anguille S, Smits EL, Lion E, van Tendeloo VF, Berneman ZN (2014) Clinical use of dendritic cells for cancer therapy. Lancet Oncol 15: e257-267.

27. Petsch B, Schnee M, Vogel AB, Lange E, Hoffmann B, et al. (2012) Protective efficacy of in vitro synthesized, specific mRNA vaccines against influenza A virus infection. Nature Biotechnology 30: 1210-6.

28. Hoerr I, Obst R, Rammensee HG, Jung G (2000) In vivo application of RNA leads to induction of specific cytotoxic $T$ lymphocytes and antibodies. Eur J Immunol 30: 1-7.

29. Pascolo S (2004) Messenger RNA-based vaccines. Expert Opin Biol Ther 4: 1285-1294.

30. Pascolo S (2006) Vaccination with messenger RNA. Methods Mol Med 127: 23-40.

31. Van Lint S, Goyvaerts C, Maenhout S, Goethals L, Disy A, et al. (2012) Preclinical evaluation of TriMix and antigen mRNA-based antitumor therapy. Cancer Res 72: 1661-1671.

32. Ceppi M, Ruggli N, Tache V, Gerber H, McCullough KC, et al. (2005) Double-stranded secondary structures on mRNA induce type I interferon (IFN alpha/beta) production and maturation of mRNA-transfected monocyte-derived dendritic cells. J Gene Med 7: 452-465.

33. Kramps T, Probst J (2013) Messenger RNA-based vaccines: progress, challenges, applications. Wiley Interdiscip Rev RNA 4: 737-749.

34. Atkins GJ, Fleeton MN, Sheahan BJ (2008) Therapeutic and prophylactic applications of alphavirus vectors. Expert Rev Mol Med 10: e33.

35. Lundstrom K (2002) Alphavirus-based vaccines. Curr Opin Mol Ther 4: 28-34.

36. Pijlman GP, Suhrbier A, Khromykh AA (2006) Kunjin virus replicons: an RNA-based, non-cytopathic viral vector system for protein production, vaccine and gene therapy applications. Expert Opin Biol Ther 6: 135-145.

37. Rayner JO, Dryga SA, Kamrud KI (2002) Alphavirus vectors and vaccination. Rev Med Virol 12: 279-296.

38. Zimmer G (2010) RNA replicons - a new approach for influenza virus immunoprophylaxis. Viruses 2: 413-434.

39. Mogler MA, Kamrud KI (2015) RNA-based viral vectors. Expert Rev Vaccines 14: 283-312.

40. Tratschin JD, Ruggli N, McCullough KC (2008) Pestivirus replicons providing an RNA-based viral vector system. EP2130912. WO 2009146867.

41. Strauss JH, Strauss EG (1994) The alphaviruses: gene expression, replication, and evolution. Microbiol Rev 58: 491-562.

42. Saeedi BJ, Geiss BJ (2013) Regulation of flavivirus RNA synthesis and capping. Wiley Interdiscip Rev RNA 4: 723-735.

43. Li SH, Dong H, Li XF, Xie X, Zhao H, et al. (2013) Rational design of a flavivirus vaccine by abolishing viral RNA 2'-O methylation. J Virol 87: 5812-5819.

44. Moser C, Stettler P, Tratschin JD, Hofmann MA (1999) Cytopathogenic and noncytopathogenic RNA replicons of classical swine fever virus. J Virol 73: 7787-7794.

45. Démoulins T, Milona P, Englezou P, Suter R, Leduc C, et al. (2015) Polyethylenimine-based polyplex delivery of self-replicating RNA vaccines. Nanomedicine (submitted for publication) 
46. Suter R, Summerfield A, Thomann-Harwood LJ, McCullough KC, Tratschin JD, et al. (2011) Immunogenic and replicative properties of classical swine fever virus replicon particles modified to induce IFNalpha/beta and carry foreign genes. Vaccine 29: 1491-1503.

47. Midoux P, Pichon C, Yaouanc JJ, Jaffrès PA (2009) Chemical vectors for gene delivery: a current review on polymers, peptides and lipids containing histidine or imidazole as nucleic acids carriers. $\mathrm{Br} J$ Pharmacol 157: 166-178.

48. Pichon C, Midoux P (2013) Mannosylated and histidylated LPR technology for vaccination with tumor antigen mRNA. Methods Mol Biol 969: 247-274.

49. Démoulins T, Milona P McCullough KC (2014) Alginate-coated chitosan nanogels differentially modulate class-A and class-B CpG-ODN targeting of dendritic cells and intracellular delivery. Nanomedicine 10: 1739-1749.

50. Geall AJ, Verma A, Otten GR, Shaw CA, Hekele A, et al. (2012) Nonviral delivery of self-amplifying RNA vaccines. Proc Natl Acad Sci U S A 109: 14604-14609.

51. Xu J, Luft JC, Yi X, Tian S, Owens G, et al. (2013) RNA replicon delivery via lipid-complexed PRINT protein particles. Mol Pharm 10: 3366-3374.

52. Démoulins T, Bassi I, Thomann-Harwood L, Jandus C, Kaeuper P, et al. (2013) Alginate-coated chitosan nanogel capacity to modulate the effect of TLR ligands on blood dendritic cells. Nanomedicine 9: 806-817.

53. Thomann-Harwood LJ, Kaeuper P, Rossi N, Milona P, Herrmann B, et al. (2013) Nanogel vaccines targeting dendritic cells: contributions of the surface decoration and vaccine cargo on cell targeting and activation. J Control Release 166: 95-105.

54. Bogers WM, Oostermeijer H, Mooij P, Koopman G, Verschoor EJ, et al. (2014) Potent immune responses in Rhesus Macaques induced by nonviral delivery of a self-amplifying RNA vaccine expressing HIV Type 1 envelope with a cationic nanoemulsion. J Infect Dis.

55. Brito LA, Chan M, Shaw CA, Hekele A, Carsillo T, et al. (2014) A cationic nanoemulsion for the delivery of next-generation RNA vaccines. Mol Ther 22: 2118-2129.

56. Ljungberg K, Liljeström P (2015) Self-replicating alphavirus RNA vaccines. Expert Rev Vaccines 14: 177-194.

57. European-Commission (2013) UniVax: A “Universal” Influenza Vaccine through Synthetic, Dendritic Cell-Targeted, Self-Replicating RNA Vaccines. Cordis Projects and Results.

58. Allard SD, De Keersmaecker B, de Goede AL, Verschuren EJ, Koetsveld J, et al. (2012) A phase I/IIa immunotherapy trial of HIV-1-infected patients with Tat, Rev and Nef expressing dendritic cells followed by treatment interruption. Clinical Immunology 142: 252-268.

59. Midoux P, Pichon C (2015) Lipid-based mRNA vaccine delivery systems. Expert Rev Vaccines 14: 221-234.

60. Su Z, Dannull J, Yang BK, Dahm P, Coleman D, et al. (2005) Telomerase mRNA-transfected dendritic cells stimulate antigen-specific CD8+ and $\mathrm{CD} 4+\mathrm{T}$ cell responses in patients with metastatic prostate cancer. J Immunol 174: 3798-3807.

61. Van Driessche A, Van de Velde AL, Nijs G, Braeckman T, Stein B, et al. (2009) Clinical-grade manufacturing of autologous mature mRNAelectroporated dendritic cells and safety testing in acute myeloid leukemia patients in a phase I dose-escalation clinical trial. Cytotherapy 11: 653-668.

62. Van Lint S, Renmans D, Broos K, Dewitte H, Lentacker I, et al. (2015) The ReNAissanCe of mRNA-based cancer therapy. Expert Rev Vaccines 14: 235-251.

63. Vik-Mo EO, Nyakas M, Mikkelsen BV, Moe MC, Due-Tonnesen P, et al. (2013) Therapeutic vaccination against autologous cancer stem cells with mRNA-transfected dendritic cells in patients with glioblastoma. Cancer Immunology, Immunotherapy 62: 1499-1509.

64. Diken M, Kreiter S, Selmi A, Türeci O, Sahin U (2013) Antitumor vaccination with synthetic mRNA: strategies for in vitro and in vivo preclinical studies. Methods Mol Biol 969: 235-246.

65. Rausch S, Schwentner C, Stenzl A, Bedke J (2014) mRNA vaccine CV9103 and CV9104 for the treatment of prostate cancer. Hum Vaccin Immunother 10: 3146-3152

66. Bernstein DI, Reap EA, Katen K, Watson A, Smith K, et al. (2009) Randomized, double-blind, Phase 1 trial of an alphavirus replicon vaccine for cytomegalovirus in CMV seronegative adult volunteers. Vaccine 28: 484-493.

67. Slovin SF, Kehoe M, Durso R, Fernandez C, Olson W, et al. (2013) A phase I dose escalation trial of vaccine replicon particles (VRP) expressing prostate-specific membrane antigen (PSMA) in subjects with prostate cancer. Vaccine 31: 943-949.

68. Wecker M, Gilbert P, Russell N, Hural J, Allen M, et al. (2012) Phase I safety and immunogenicity evaluations of an alphavirus replicon HIV-1 subtype $\mathrm{C}$ gag vaccine in healthy HIV-1-uninfected adults. Clin Vaccine Immunol 19: 1651-1660.

69. Sung H, Schleiss MR (2010) Update on the current status of cytomegalovirus vaccines. Expert Rev Vaccines 9: 1303-1314. 